\title{
The impact of 'negative data'
}

\author{
Vinod Labhasetwar
}

Published online: 17 December 2014

(C) Controlled Release Society 2014

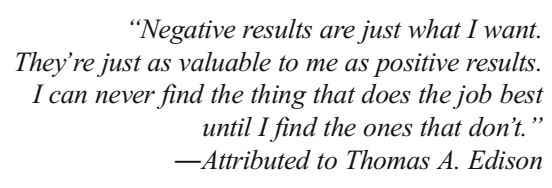

There is an ongoing debate on the value of publishing (and the effects of withholding) negative research results. I discussed this very point during the meeting of the editorial board for Drug Delivery and Translational Research (DDTR), held during the 41st annual meeting of the Controlled Release Society in Chicago this past July. There, the board members agreed that DDTR should consider manuscripts reporting negative data, as long as the research is based on a strong rationale or hypothesis.

We all know that neither all experiments lead to expected or positive results nor are all proposed hypotheses correct. Oftentimes, we encounter more failure than success, but we report only positive results simply because it is hard to publish negative results. I can give you an instance from my own experience. The study was part of a peer-reviewed, federally funded grant carried out about 20 years back. The experiment was to test a time-release capsule following oral delivery. We tested this capsule in rabbits simply because we thought we could easily draw blood multiple times in sufficient quantities through an ear vein to analyze drug levels to show timerelease drug profile. The other reason was that we could easily feed a regular-size capsule used in humans to rabbits, which for a study in rats would have required miniaturizing the size.

Why did this experiment fail? This is because gastric emptying time in rabbits is significantly longer than in rats or humans. The rabbit stomach is like a bag that can retain a significant amount of food, even after $24 \mathrm{~h}$ fasting. Prolonged

V. Labhasetwar $(\triangle)$

Biomedical Engineering, Lerner Research Institute, Cleveland

Clinic, Cleveland, OH 44195, USA

e-mail: labhasv@ccf.org gastric retention time is necessary because rabbits are herbivores; their diet contains large amounts of cellulose, which is hard to digest. Our capsule had been designed to withstand gastric $\mathrm{pH}$ for a few hours and then open up in the small intestine at a different time interval. In this experiment, we could detect the intact capsule retained in the rabbit stomach even after one full day. Since the capsule was retained in the acidic $\mathrm{pH}$ for so long, it failed to function as expected. The important lesson to be learned, for our own and others' investigations, was that the rabbit is not the best animal model in which to test oral time-release or sustained drug delivery systems. Yet we could not publish the study results because of the "negative" outcome.

While our own DDTR will retain its main purpose as a vehicle for all translational aspects of drug delivery, we of the Editorial Board recognize that our readers could benefit from the publication of negative results and from reports of what approaches did not work as expected. If methodological or logistical flaws of a research path are quickly brought to light, other investigators may not continue down that same ineffectual path, and in the end, funding and valuable laboratory resources will be better used. If one group reports negative findings, there may be a useful learning curve for us all based on that negative data so that others might get novel ideas in terms of revising their hypotheses or experimental designs. For some, negative data from one study could provide a rationale or new approach/paradigm to pursue. It is well known that many experiments work well in vitro but not in vivo or fail to demonstrate efficacy in preclinical testing. Unexpected toxicity may limit the benefits of a drug or a particular mode of delivery. Reporting negative data from 
clinical trials is clearly essential, as it can have a direct impact on human health.

From now on, DDTR will consider manuscripts that report negative results. Such manuscripts will undergo the standard, rigorous review process, but the reviewers will be alerted to the nature of the manuscript. Authors are strongly encouraged to discuss the possible reasons why experiments failed and what alternative approaches or changes to the hypothesis or experimental design might be made. A cover letter to the editor briefly describing the above points will be helpful.

We expect that this new editorial policy will enhance DDTR, making it an improved tool for all of us in the drugdelivery field. We welcome your views, pro or con, and look forward to hearing from you. 